\title{
Déterminants définis et noms inaliénables en position objet en français de Côte d'Ivoire
}

\author{
Boutin, Béatrice Akissi \\ ILA-UFHB, Abidjan-Cocody \\ La Sapienza, Roma \\ Version auteur (mise en page des exemples garantie)
}

p. 148

Nous souhaitons dans cette contribution à un ouvrage sur la variation du français dans les espaces francophones nous pencher sur l'expression de la possession inaliénable en position de complément verbal direct en français de Côte d'Ivoire. Il s'agira de comparer les constructions avec un pronom réfléchi à valeur de datif de totalité (Riegel 1991) ou datif possessif (Lamiroy \& Delbecque 1997): il se lave le visage, et les constructions avec un déterminant possessif associé à un nom de partie du corps : il lave son visage. Pour traiter cette question, nous reprendrons plusieurs analyses du nom inaliénable en position objet en français et dans d'autres langues (1), nous verrons ensuite le décalage entre le français de référence et le français de Côte d'Ivoire et nous rappellerons le statut et les emplois du déterminant défini selon la norme ivoirienne (2). Enfin, nous enrichirons la description grammaticale de la possession inaliénable avec des données métalinguistiques recueillies auprès d'adolescents, sur des phrases qu'ils jugent ou auxquelles ils proposent un équivalent (3).

Le contexte ivoirien nous permet de considérer l'actualisation de plusieurs normes de français, tant à l'écrit, qu'à l'oral, que dans le cadre particulier de l'institution scolaire. En effet, il n'est pas rare d'observer des pratiques plurilingues et des passages d'une norme de français à l'autre chez les élèves et les enseignants au sein même de la classe (Kouamé 2013). Nous nous appuierons essentiellement sur un corpus ancien (2002, littérature et presse écrite), et sur un corpus récent (2018) constitué des réponses de collégiens à deux items de deux Tests des habiletés métalinguistiques («Acceptabilité » et «Ambiguïté »du THAM 2, Pinto et al. 2015). Notre approche est donc à la fois syntaxique et cognitive.

\section{Les analyses syntaxiques du nom inaliénable en position objet}

Nous nous intéressons tout spécialement aux analyses des phrases de structure N0 (se) V (Ddéf/Dposs) N1 dans lesquelles le complément direct N1 est un nom inaliénable coréférent au sujet N0, et aux variantes concernant le

p. 149

déterminant de N1. Nous commencerons par les études menées dans le cadre de la grammaire transformationnelle, et nous les complèterons par d'autres qui éclairent certains aspects sémantiques et cognitifs ${ }^{1}$.

Guéron (1983) étudie, en français de référence ou européen (Fréf), différents cas où un déterminant défini de type le, la, les introduit un nom de référent inaliénable et avance, dans le cadre de l'école générative, plusieurs conditions pour que le nom ait cette interprétation. Notamment, le déterminant défini doit être présent comme une anaphore de l'antécédent N0, le nom correspondant au possesseur, N0 devant donc précéder dans la phrase le nom du référent inaliénable.

\footnotetext{
${ }^{1}$ Notre compte-rendu de ces analyses ne peut être ici que partiel ; nous citons les auteurs pour leurs apports principaux à la discussion, mais les choix que nous faisons pour citer un auteur plutôt qu'un autre pour un aspect de la discussion ne signifient pas que les autres n'aient pas abordé cet aspect.
} 
D'autres facteurs interviennent pour que certaines phrases soient interprétées avec la relation inaliénable et d'autres non. De nombreux verbes transitifs empêchent une interprétation inaliénable de $\mathrm{N} 1$, pour les mêmes noms de partie du corps :

Fréf : (1a) 1. Jean a levé les yeux, bougé la tête, donné la main, avancé le pied, crispé les poings, etc. 2.* Jean a rincé les yeux, palpé la tête, massé le pied, blessé les poings, épilé les sourcils, etc. (J. Guéron 1983 : 29-30).

Les verbes lever, bouger, donner, avancer, crisper dénotent, pour Guéron (1983), des « gestes naturels simples » alors que les gestes dénotés par les verbes rincer, palper, masser, blesser, épiler ne le sont pas. Pour ce deuxième type de verbes, Guéron 1983 considère que le pronom préverbal se est nécessaire pour jouer le rôle d'antécédent et favoriser l'interprétation inaliénable du nom qui suit. Nous avons alors pour ces phrases :

Fréf : (1b) 1. Jean s'est rincé les yeux, palpé la tête, massé le pied, blessé les poings, épilé les sourcils

L'auteure distingue donc deux types de phrases : celles construites avec le pronom préverbal se, qui correspond au nom possesseur (en référence à M. Gross (1968:31-34)), et celles construites sans ce pronom :

Fréf : (1c) 1.Il $(E / *$ se) lève les yeux

2. Il (*E/se) blesse les poings (Guéron $1983: 24)$.

p. 150

On remarque que l'emploi de se est impossible pour les verbes de la première classe s'ils sont suivis d'un complément :

Fréf : (1d) 1. * Jean s'est levé les yeux, bougé la tête, donné la main, avancé le pied, crispé

les poings, froncé les sourcils, etc.

Avec le pronom se, ces verbes ne peuvent entrer que dans des constructions intransitives :

Fréf : (1e) 1. Jean s'est (levé / bougé / donné / avancé / crispé).

Pour les verbes de la deuxième classe, l'emploi de se est indépendant de la présence d'un nom de partie du corps en position objet en français de référence. On obtient donc la synthèse suivante de ces deux classes de verbes très différents, utilisés avec le pronom se :

Fréf : (1f) 1. Jean a (levé / bougé / donné / avancé / crispé) (*E / Npc)

1. Jean s'est (levé / bougé / donné / avancé / crispé) (E / *Npc)

2. Jean a (rincé / palpé / massé / blessé / lavé)*(E/Npc)

2. Jean s'est (rincé / palpé / massé / blessé / lavé) (E / Npc).

Lamiroy \& Delbecque (1997) étudient minutieusement la deuxième classe de verbes dans plusieurs langues romanes et germaniques. Elles se penchent particulièrement sur la valeur de datif possessif du pronom préverbal se. Se représente le possesseur, ou l'entité totale dont l'un des éléments de sa sphère personnelle est affecté par le procès Les auteures suivent la tradition de Bally (1926) sur la sphère personnelle, qui comprend d'abord les noms de parties du corps, puis les termes de parenté et enfin jusqu'à certains objets possédés. Elles soulignent que dans ces constructions, le datif possessif est particulièrement impliqué dans le procès au point de fonctionner comme un réel argument. Cela le distingue du datif éthique qui reste un témoin extérieur au procès.

Une fois repérées les deux classes de verbes qui partagent la construction N0 (se) V N1 dans lesquelles le complément direct $\mathrm{N} 1$ est un nom inaliénable coréférent au sujet N0, elles peuvent être analysées conjointement en ce qui concerne le déterminant du nom inaliénable. Nombre 
d'auteurs affirment que le déterminant possessif est rarement utilisé dans ces structures avec un nom inaliénable en français de référence. Par exemple, les phrases libres :

Fréf : (2a) Guy se lave les mains
Va te brosser les dents

sont estimées plus naturelles ou courantes que :

(2b) Guy lave ses mains

p. 151

Va brosser tes dents.

De même que les phrases figées :

Fréf : (3a) Max se rafraîchit la mémoire

Max se ruine la santé

par rapport à :

(3b) * Max rafraîchit sa mémoire

* Max ruine sa santé (M. Gross 1998 : 2, 34).

$\mathrm{Au}$ contraire, le déterminant possessif est préféré avec un nom aliénable :

(4) Guy lave sa casquette, brosse son cheval, ruine sa famille.

Avec un nom inaliénable, le déterminant possessif n'est utilisé que si celui-ci n'est pas coréférent au sujet :

Fréf : (5a) Guy lave les mains de Eve / Guy lave ses mains

Guy rafraîchit la mémoire de Eve / Guy rafraîchit sa mémoire

Guy ruine la santé de Eve / Guy ruine sa santé.

Cependant, même dans ce cas, la construction avec un pronom préverbal datif bénéfactif et un déterminant défini est préférée en français de France (M. Gross 1998 : 32-35) :

Fréf : (5b) Guy lui lave les mains

Guy lui rafraîchit la mémoire

Guy lui ruine la santé.

Riegel (1991) ne partage pas l'analyse classique pour les langues romanes dans laquelle le déterminant défini « exprim[e] le rapport de "possession inaliénable" qui lie le nom de la partie au nom du tout », ni l'analyse comme un marqueur d'anaphore au sens de Chomsky ou Guéron. Pour l'auteur, si le déterminant défini est anaphorique, c'est dans le sens que le possesseur est facilement récupérable dans l'information contextuelle à partir du moment où les locuteurs tiennent pour acquis qu'un tout est affecté en même temps que sa partie. Son analyse du déterminant défini pour les noms inaliénables repose sur l'analyse de la transitivité des verbes qui y sont impliqués.

Riegel (1991) étudie des phrases à construction transitive dans lesquelles le procès affecte à la fois un tout animé et une partie constitutive de ce tout. Il soulève, à propos de ces phrases, plusieurs questions sur la grammaticalisation de certains principes cognitifs. Nous retenons ici celle de la solidarité des

p. 152

parties au tout animé, à l'intérieur de la sphère personnelle, expression qu'il emprunte lui-aussi à Bally (1926). Dans les exemples suivants, vous et lui sont grammaticalement des compléments datifs et sémantiquement le tout animé dont la partie est affectée, affecté lui-aussi conjointement. Par ailleurs, si le tout n'est pas animé (le téléphone), la construction est étrange.

Fréf : (6) Qui vous a coupé les cheveux?

? Qui a coupé vos cheveux?

? Qui lui a coupé le fil (au téléphone)?

Je vais lui tirer les oreilles (Riegel 1991 : 133, 137) 
Pour l'auteur, le complément datif de la totalité engage la personne (dans sa totalité) dans un procès qui demande au moins son «contrôle vague ». Dans le cas contraire, avec des verbes qui n'impliquent pas le contrôle du sujet, la construction avec le datif de totalité et le déterminant défini n'est pas possible; seul le déterminant possessif est possible :

Fréf : (7) Pierre lui brosse/casse/soigne les dents

*Pierre lui admire/voit connait les dents (Riegel 1991 : 138)

Pierre admire/voit connait ses dents.

Dans les deux cas cependant, le verbe est transitif et se construit normalement avec un seul argument en position objet (ici le N1 de N2). Au plan grammatical, un dédoublement de cet argument en un objet (N1 la partie du corps) et un pronom datif de totalité (pour de N2) est possible, lorsque le possesseur exerce un certain contrôle sur le procès.

L'analyse de Junker \& Martineau (1987) des possessions inaliénables en position objet, cette fois coréférentes au sujet, est éclairante sur plusieurs aspects, notamment sémantiques. Deux propriétés essentielles des noms inaliénables susceptibles d'entrer dans une construction objet avec déterminant défini sont mises en lumière. D'abord, les auteures explicitent la notion d'inaliénabilité en mettant en avant la propriété d'inclusion qui permet de faire une distinction fondamentale entre les constructions avec le déterminant possessif, qui correspondent à une interprétation possessive d'appartenance, des constructions avec le déterminant défini qui correspondent à une interprétation d'inclusion en français de référence.

«Cette relation d'inclusion est préconstruite dans nos schémas conceptuels. C'est notre connaissance du monde, du domaine extra-linguistique, qui nous permet d'évaluer la relation existant entre les entités [...] l'inclusion se distingue de l'appartenance en ce que la première est comprise a priori comme une relation inaliénable entre

p. 153

deux objets : la partie du corps SN2 est consubstantielle de SN1 qui est le tout. » (Junker \& Martineau 1987 : 198)

Cependant, alors que cette connaissance du monde et l'antéposition de N0 pourrait justifier le rôle anaphorique du déterminant défini, les auteurs pointent que l'interprétation du nom comme inaliénable, ou inclus, peut ne pas reposer sur le déterminant défini et ses propriétés anaphoriques, puisque d'autres déterminants entrent dans la même construction :

Fréf : (8) 1. Il a levé un pied/ une main

2. Edga se lava soigneusement une oreille, puis l'autre ${ }^{2}$ (Junker \& Martineau 1987) Pour les auteures, la différence essentielle réside plutôt entre les deux classes de verbes : celle qui demandent un réfléchi et celle qui ne le demandent pas pour que le N1 objet, précédé du déterminant défini, puisse être interprété comme une partie du corps de N0.

Fréf: (9) 1. Jean lève la tête

2. Jean se brosse les dents (Junker \& Martineau 1987)

Après avoir montré par divers tests (passivation, modification par un adjectif) l'équivalence sémantique des deux constructions, les auteurs mettent en évidence que la différence réside dans les propriétés attribuées au référent du nom inaliénable ou inclus en regard au verbe : s'il est admis qu'il ne peut réaliser l'action dénotée par le verbe « de lui-même », le datif est obligatoire avant le verbe :

Fréf : (10) Elte bouge les yeux/la main/la tête/*les cheveux (Junker \& Martineau 1987)

La main/la tête de Elte bouge d'elle-même /

Les yeux/*les cheveux bougent d'eux-mêmes

${ }^{2}$ C'est nous qui rajoutons 1 et 2, en écho aux deux classes de Guéron (1983) vues plus haut. 
Elte se bouge les cheveux.

Le pronom préverbal se n'a donc pas seulement un rôle d'antécédent, mais il indique une implication du sujet plus importante que dans les constructions sans se. Cette propriété, qui se vérifie par un test du manque d'autonomie du nom de partie du corps (10), est plus convaincante que la simplicité du geste de Guéron (1983) et elle complète le contrôle du procès par le sujet de Riegel (1991).

p. 154

Ces propriétés sémantiques des noms corrélées à des propriétés syntaxiques se retrouvent de façons diverses dans les langues romanes et germaniques comme nous le verrons plus bas à travers d'autres auteurs. La notion même d'inaliénabilité doit d'abord être détaillée.

Simatos (2015) s'intéresse, entre autres, aux limites de la classe de noms inaliénables précédés d'un déterminant défini, qui peuvent entrer dans une construction dative en français de référence. L'auteure s'appuie sur la contrainte de partage du rôle sémantique entre le datif de totalité et le nom inaliénable. En plus des noms de parties du corps qui sont prototypiques, trois principaux types de noms « essentiels », ou constitutifs, abstraits peuvent être utilisés dans une construction avec un datif obligatoire :

- Noms d'organes abstraits : p. ex. âme, cour, esprit...

- Noms d'aspects des individus : p. ex. allure, apparence, caractère, comportement, constitution, humeur, nature, personnalité, tempérament...

- Noms de puissances humaines : p. ex. appétit, digestion, imagination, jugement, marche, mémoire, moral, parole, pensée, raison, respiration, santé, sommeil, vie, voix, vue...

Ces limites sont mises en évidence par la possibilité d'entrer dans la construction avec le datif et le déterminant défini en français de référence.

Fréf : (11) Il y a quelque chose qui (*E/lui) a (troublé la mémoire/ perturbé le sommeil/ gêné la respiration/ calmé l'esprit/ rempli l'imagination (Simatos 2015 : 134-135)

Au niveau de la diversité des langues, la question de la concurrence entre la construction avec le déterminant possessif et celle avec le datif mais sans le possessif/génitif revient à une question sur l'existence d'un traitement particulier de la possession inaliénable en position d'objet.

Lamiroy (2001), dans une analyse fine de trois langues romanes et trois langues germaniques, teste l'hypothèse d'une grammaticalisation vers « un nouveau schéma actantiel qui se fait au détriment de la zone du datif et donne davantage de saillance à la zone du sujet/nominatif » (Lamiroy 2001 : 94). Elle explique ainsi la préférence de certaines langues européennes pour la construction avec le datif possessif et le déterminant défini par rapport à celle avec le génitif ou le déterminant possessif. Le néerlandais, par exemple, apparait comme une langue qui anciennement acceptait plus librement la construction avec le datif et le déterminant défini qu'aujourd'hui, où cette construction ne subsiste presque que dans des expressions figées. En anglais le processus aurait complètement abouti puisque le datif possessif est absent.

*I wash me the hands

p. 155

I wash my hands (Lamiroy $2001: 89$ )

Le français de référence a des critères beaucoup plus restrictifs que les autres langues romanes pour construire des phrases avec le datif et le déterminant défini. Par exemple, cette construction est obligatoire pour les noms de partie du corps alors que les noms de parenté sont moins admis dans cette construction (Voir aussi (3) et (5)).

(13)
Italien
Il medico ha esaminato loro la gola
Espagnol
El médico les examinó la garganta
Fréf
Le médecin leur a examiné la gorge 
?* Le médecin a examiné leurs gorges (Lamiroy $2001: 5$ )

\section{(14) Espagnol Se les casa la última hija mañaña \\ Fréf $\quad *$ La dernière fille se leur marie demain \\ Leur dernière fille se marie demain (Lamiroy 2001 : 10)}

Les divers comportements des langues étudiées correspondent pour l'auteure à une échelle de grammaticalisation, dans laquelle la primauté est donnée de plus en plus au sujet/nominatif au détriment du complément datif. Lamiroy (2001) ne mentionne pas dans cette hypothèse la répercussion sur la perte du traitement particulier de la possession inaliénable opéré par le datif. Dans la théorie de l'agentivité suivie par l'auteure, la concurrence entre ces deux actants se solde en diachronie par le seul maintien du nominatif et au détriment du datif.

Au contraire, Creissels (2006), qui étudie la concurrence des deux constructions sur un important nombre de langues de divers lieux et familles, ne voit aucun argument pour orienter le processus de grammaticalisation vers la construction avec le génitif plutôt que vers la construction avec le datif. Pour lui, l'anglais est un cas typique des langues qui tendent à utiliser systématiquement les génitifs et possessifs, et les langues romanes ou le basque des cas typiques des langues qui ont tendance à se passer des génitifs ou possessifs surtout pour les référents très proches du sujet humain. Cette tendance est plus forte en occitan, en espagnol et en italien qu'en français, mais c'est en roumain qu'elle atteint son degré extrême, proche en cela du basque. Nous remarquons aussi qu'il n'y a pas de corrélation entre la typologie, la génétique ou la géographie des langues.

p. 156

Creissels (2006) s'oppose à prendre comme modèle plus abouti, ou au contraire plus primaire, l'un ou l'autre de ces types de constructions. Dans la citation suivante, il se réfère respectivement à la construction avec le génitif ou le déterminant possessif et à celle avec le datif et le nom sans morphème génitif :

« [L]orsqu'une entité B se trouve impliquée dans un événement par l'intermédiaire d'un élément A de sa sphère personnelle, il n'est pas correct de considérer a priori comme plus 'naturelle' l'une ou l'autre des deux solutions consistant, soit à traiter le constituant nominal qui se réfère à $\mathrm{B}$ comme génitif (laissant ainsi implicite son rôle de bénéficiaire ou détrimentaire dans l'événement que dénote le verbe), soit à traiter $\mathrm{B}$ comme argument du verbe (laissant ainsi implicite son statut de possesseur relativement à un autre participant). » (Creissels 2004, chapitre 16, p. 12)

Par ailleurs, selon l'auteur, aucune distinction tranchée ne peut être établie à partir des faits linguistiques entre des entités inaliénables que la langue traite comme directement impliquées aussi dans l'événement réalisé par le sujet humain et dénoté par le verbe, et des entités aliénables, traitées comme ne participant pas, mais qui sont dans des relations de type possessives avec les participants.

A la fin de cette discussion sur les langues européennes, nous relevons premièrement que la classe des noms possédés et inaliénables entrant dans une construction avec le datif et le déterminant défini diffère selon les langues, qu'on peut observer un manque de cohérence au sein d'une même langue, et que certaines langues ne font aucune distinction basée sur la notion d'inaliénabilité. Par exemple, les langues romanes du sud, tout comme le français méridional, étendent largement les noms susceptibles d'entrer dans la construction avec le datif et un déterminant non possessif, bien au-delà des parties du corps et de la sphère « inaliénable ».

$$
\begin{array}{ll}
\text { français méridional } & \text { mets-toi le chapeau } \\
\text { espagnol } & \text { ponte el sombrero }
\end{array}
$$


italien

mettiti il cappello

Deuxièmement, nous retenons que les langues traitent différemment l'implication des entités inaliénables dans les événements qui les affectent en même temps que le sujet total.

Troisièmement, il nous semble qu'un rôle sans doute trop important est accordé au déterminant défini. En effet, si le nom de partie du corps est interprété comme rattaché à la personne totale, ce processus ne s'appuie pas forcément sur une valeur anaphorique en contexte du déterminant défini p. 157

mais surtout sur la connaissance du monde des interlocuteurs. La principale distinction serait donc entre les constructions avec un datif argument du verbe et les constructions avec un génitif associé au nom. Pour étayer cette hypothèse, nous avons, en marge des constructions analysées jusque-là (Voir aussi (8)), d'autres constructions dans lesquelles aucun déterminant n'apparait après certaines prépositions comme :

(16a) Guy est venu sans voiture

Guy a 30 euros en poche

Dans ces phrases, l'interprétation de la voiture ou de la poche comme possessions (aliénables) de Guy semble évidente (Guy a une voiture, une poche). Elle n'est pourtant pas automatique et ne dépend pas de la préposition. Dans la phrase suivante, interpréter la voiture comme celle de Guy (Guy a une voiture ?) est beaucoup moins clair :

(16b) Guy est venu en voiture.

Dans les constructions avec le datif, c'est le nom inclus ou inaliénable lui-même (ou même aliénable dans certaines langues (15)), plus que le déterminant défini qui induit le rattachement de l'objet au nom de la personne totale déjà présent.

Dans ce sens, Kpli (2014) pose, au sein de la théorie métaopérationnelle, que dans les constructions avec le datif, le procès est déjà orienté vers le sujet, ce qui rend prioritaire ou évidente l'interprétation du nom inaliénable comme rattaché à ce sujet. Le défini le, déterminant utilisé pour les noms évidents en discours, est justifié par le caractère prédéterminé, présupposé et acquis de la relation d'appartenance entre le nom de personne et le nom de partie du corps (Kpli 2014 : 6). Le déterminant possessif (ou un génitif dans les langues sans déterminant possessif) serait redondant avec un nom inaliénable puisque l'existence des parties du corps de la personne est déjà acquise à partir de l'actualisation du nom sujet. Pour l'auteur, la notion de possession inaliénable se définit comme une relation d'appartenance dans laquelle l'élément possédé ne peut être séparé du possesseur. Ce caractère inséparable de la relation possesseur-possédé s'exprime en syntaxe de manière particulière.

A la suite, Kpli (2014) oppose des langues qui orientent l'interprétation à partir du nom humain vers ses parties du corps (tout nom humain a nécessairement des parties de corps), et des langues qui posent principalement que tout nom de partie de corps est nécessairement de quelqu'un. Au premier type appartiennent les langues romanes, le basque, les langues germaniques, y compris l'anglais qui permet quelques cas avec le déterminant défini :

\section{(17) She patted him on the head}

p. 158

*She patted him on his head. (Cooper (2002 : 2), cité par Kpli 2014)

Au second type appartient, par exemple, le baoulé de Côte d'Ivoire. Dans cette langue, un nom de partie du corps ne peut être réalisé sans référence à un nom humain ou animé : le nom de personne s'il est actualisé en discours, ou le pronom bé (eux, quelqu'un, on) si le nom de partie du corps est désigné seul, comme notion : bé tí (tête de quelqu'un, d'eux), bé sá (bras de quelqu'un, d'eux). Au 
contraire, une notion hors des parties du corps (possession aliénable) pourra être citée seule sans cette contrainte. L'auteur donne en exemple àsic (sol) et waka (arbre).

Lorsque le nom de partie du corps est actualisé en discours, il le sera toujours avec un génitif :
(18) baoulé
$\boldsymbol{m i} \boldsymbol{i}$ ' $\boldsymbol{n}$ yo
$m \tilde{\imath}$
$y a$
1SG tête-DEF fait P1 mal
(yo...ya : faire mal)
(la mienne tête fait à moi mal)
j'ai mal à la tête (Tymian et al 2003 : 470, voir aussi Kpli 2014 : 6)

La redondance est largement acceptée dans la langue baoulé, et un pronom de reprise comme $\boldsymbol{i}$ dans (19) figure souvent entre le nom et le complément du nom dans un syntagme génitival, qui a pour ordre génitif - non tête :

(19) baoulé
be kpeli labu'n i ti'n
3PL.NOM couper-ACC canard-DEF P3 tête- DEF
(on a coupé la tête de lui le canard)
on a coupé la tête du/au canard (Tymian et al 2003 : 424)

L'analyse de Kpli (2014) est d'autant plus intéressante qu'elle montre que le génitif est indispensable pour exprimer la possession inaliénable dans certaines langues, à l'opposé des analyses présentées jusqu'ici.

Par ailleurs, comme le fait remarquer Kpli (2014), et les ethno-psychologues/thérapeutes le confirment, la représentation mentale de l'événement est très différente dans j'ai mal à la tête et ma tête me fait mal. Dans le premier cas le sujet porte le poids de l'événement, alors que dans le deuxième cas, « il s'agit d'isoler la partie du corps qui est en cause pour faire porter sur elle tout le poids de ce que l'énonciateur veut exprimer, c'est-à-dire la douleur » (Kpli 2014: 6) et un dialogue s'instaure entre la partie du corps

p. 159

et la personne ${ }^{3}$. Néanmoins, la relation possesseur possédé est bien explicitée.

De la pratique des langues ivoiriennes de toujours faire figurer le génitif aux côtés du nom inaliénable, Kpli (2014) fait découler les énoncés mon pied me fait mal, ma tête me fait mal du français de Côte d'Ivoire (18). On peut ajouter plusieurs autres exemples baoulé dans lesquels le nom inaliénable est en position objet obligatoirement avec le génitif. Ces constructions éclairent efficacement les constructions correspondantes en français de Côte d'Ivoire.

(20) baoulé tra wó nuan nun; man wó sá su; fa wó tààmán fite saisir 2SG bouche dedans ; lever 2SG main dessus ; prendre 2SG langue sortir

Fréf ouvre la bouche ; lève la main ; ; tire la langue

FCI ouvre ta bouche ; lève ta main ; tire ta langue

\begin{tabular}{|c|c|c|c|c|c|c|c|c|c|c|c|c|c|}
\hline (20) & baoulé & tra & wó & nuan & nun; & $\operatorname{man}$ & wó & sá & su ; & $f a$ & wó & tààmán & fite \\
\hline & & saisir & $2 \mathrm{SG}$ & bouche & dedans; & lever & $2 \mathrm{SG}$ & main & dessus & prendre & $2 \mathrm{SG}$ & langue & sortir \\
\hline & Fréf & \multicolumn{4}{|c|}{ ouvre la bouche } & \multicolumn{4}{|c|}{ lève la main; } & \multicolumn{4}{|c|}{ tire la langue } \\
\hline & FCI & \multicolumn{4}{|c|}{ ouvre ta bouche; } & \multicolumn{4}{|c|}{ lève ta main; } & \multicolumn{4}{|c|}{ tire ta langue } \\
\hline
\end{tabular}

\section{Le nom inaliénable en position objet en français de Côte d'Ivoire}

Le français de Côte d'Ivoire regroupe des mouvements opposés que nous allons tenter d'éclaircir. D'une part dans la langue littéraire surtout, on relève de larges emplois du déterminant défini avec des noms désignant des référents (in)aliénables qui seraient peu communs en français de référence, d'autre part, hors de la langue soutenue, on constate une nette préférence pour la construction avec le possessif, dans les cas où la construction avec le déterminant défini et le

\footnotetext{
${ }^{3}$ Communication personnelle de feu Annick Boutin (octobre 2019), alors logothérapeute.
} 
pronom préverbal se est, au contraire, plus courante en français de référence. Tous les faits présentés dans cette section s'éloignent du français de référence. Ils ne doivent pas occulter que les faits conformes au français de référence sont cependant les plus nombreux chez les auteurs cités et dans la presse.

a) Extension du déterminant « défini » avec un nom désignant un référent « inaliénable »

Une première remarque concerne certaines phrases figées dans lesquelles le datif $\boldsymbol{s} \boldsymbol{e}$ est nécessaire en français de référence pour que le $\mathrm{N} 1$ avec un déterminant défini soit interprété comme rattaché à NO :

p. 160

(21) Fréf Guy se retrousse les manches, se serre la ceinture.

Ces phrases ont pour variante, dans la littérature (en l'occurrence Un nègre à Paris de Bernard Dadié) et la presse de Côte d'Ivoire, une version sans le datif se :

FCI (22) L'essentiel pour l'instant est de prendre hardiment position sur des problèmes cruciaux, de retrousser les manches et de bâtir rapidement la nouvelle société. (Nèg 117)

(23) On voit bien que les princes qui nous gouvernent s'engraissent et on demande à la population de serrer la ceinture. [...] L'augmentation des prix signifie que la population seule est appelée à serrer la ceinture. (Notre Voie 17.09.1999).

La deuxième remarque concerne les phrases libres, dans lesquelles l'emploi du déterminant défini est étendu, en français de Côte d'Ivoire, à des cas plus difficiles en français de France, par exemple dans les phrases suivantes des écrivains Ahmadou Kourouma et Micheline Coulibaly. De (24) à (26), l'objet est un nom de partie du corps ou constitutif ; en (27) et (28), l'objet est une possession.

FCI (24a) Sans que l'un ou l'autre acceptât de venir en supplicié offrir l'occiput aux dents vengeresses du vieillard. (Mon 179)

(25a) Le double, le dja de Fama avait quitté le corps pendant le sommeil. (Sol 119)

(26a) A l'étranger [...] je demandais le nom totémique. (Mon 162).

(27a) Le patriarche [...] se fit ajuster le turban (Mon 221)

(28a) Il y eu des hommes fâchés d'avoir dépassé l'âge de faire des folies, d'autres mécontents d'avoir le compte en banque trop famélique pour en faire. (Emb 46).

En français de référence, ces noms sont, de préférence, précédés du déterminant possessif :

Fréf (24b) Sans que l'un ou l'autre acceptât de venir offrir son occiput aux dents du vieillard.

(25b) L'âme de Fama avait quitté son corps pendant son sommeil.

(26b) A l'étranger je demandais son nom totémique.

(27b) Le patriarche se fit ajuster son turban

(28b) Des hommes mécontents d'avoir (un / leur) compte en banque trop famélique.

p. 161

L'oral actuel confirme cette construction en français de Côte d'Ivoire :

(29) Je vous invite une fois de plus encore à laver les mains régulièrement avec de l'eau et du savon (@Lemediaivoirien, twitter, 30 avril 2020) ${ }^{4}$

Une extension de l'emploi du déterminant défini concerne aussi des phrases figées ou des combinaisons fréquentes dans lesquelles le déterminant possessif est attendu en français de référence, comme :

Fréf (30a) Guy passe son temps à dormir, tire son chapeau à Yves, regarde sa montre.

\footnotetext{
${ }^{4}$ Extrait du clip 'Lutte contre le Covid19’ réalisé avec les paroles de plusieurs influenceurs ivoiriens.
} 
En français de Côte d'Ivoire, le déterminant qui précède le complément de ces expressions figées n'est pas soumis aux mêmes contraintes ; il peut varier au profit du déterminant défini comme le montrent les séquences suivantes de la presse et de Bernard Dadié :

FCI (30b) Les étudiants ont passé le temps à se livrer à des invectives. (NV 21.12.1998)

(30c) Tous les observateurs ont tiré le chapeau aux membres du comité. (NV 04.01.1999)

(30d) Tiens, les voilà qui regardent la montre, courent, sautent du bus. (Nèg 94).

Dans une tentative d'explication de ces emplois du déterminant défini pour un nom (in)aliénable en position objet, on peut envisager que des écrivains ivoiriens comme Ahmadou Kourouma jouent avec cette construction française et l'étendent. Nous devons aussi rapprocher tous ces exemples de nombreux autres cas de l'extension d'emploi du déterminant défini (hors de la problématique du nom inaliénable en position objet) repérée par Kouadio N'Guessan 1977, 1999, Boutin 2002, Jabet 2003, Prignitz 2006 et passim, en Côte d'Ivoire et au Burkina Faso limitrophe. Pour Kouadio N'Guessan (1999), le déterminant défini se substitue fréquemment au possessif ou au partitif du français de référence, exprimant prioritairement la valeur spécifique ou circonscrite.

«On remarque un fait qui semble sous-jacent à tous ces emplois : c'est l'idée que dans la pensée du locuteur, ces noms renvoient à des référents précisément délimités (Manessy $1994 b)^{5}$. Cette valeur sémantique semble primer sur les valeurs annexes, mais

p. 162

indispensable en français central, que véhiculent les déterminants. » (Kouadio N'Guessan 1999 : 305)

Si l'auteur privilégie cette valeur spécifique plus qu'une valeur anaphorique (Riegel 1991) ou d'évidentialité (Kpli 2014) communément attribuée au déterminant défini en français, c'est parce qu'en baoulé (et dans d'autres langues ivoiriennes), les valeurs des déterminants reposent principalement sur l'opposition générique vs. spécifique (Creissels \& Kouadio N'Guessan (1977 : 296-315), Boutin (2002: 248-249)). Ce texte n'est pas le lieu d'approfondir cette question, mais nous retenons que le mécanisme particulier d'une interprétation possessive ou inclusive n'est pas forcément activé à partir du déterminant défini des compléments inaliénables en objet.

\section{b) Extension de l'emploi du déterminant « possessif »}

Hors du français académique, et parallèlement à l'extension d'emploi du déterminant défini vue précédemment, on remarque, en Côte d'Ivoire, une préférence pour la structure avec le déterminant possessif son / leur sur la structure avec le déterminant défini et éventuellement le datif se pour les noms aliénables objets. Nous avons, par exemple, les possibilités (31)-(33) en phrases libres, et un exemple oral correspondant à (31) :

FCI (31) Awa se lave les mains / Awa lave ses mains

(32) Va te brosser les dents / Va brosser tes dents

(33) Awa se pommade les pieds / Awa pommade ses pieds.

(34) Ces kits euh nous allons les mettre euh à bord de nos véhicules, afin que tous les passagers puissent laver leurs mains et protéger... parce que en nous protégeant, en protégeant tous nos conducteurs, nous protégeons aussi les passagers et nous protégeons nos familles. (03/05/2020, JT 12h30, conducteur de taxi communal, Yopougon, https://www.youtube.com/watch?v=hUQ_pgW_pHY, 3'40)

Les phrases figées du français de référence vues plus haut (3), (5), (11) ont, en français de Côte d'Ivoire, la double possibilité de construction :

${ }^{5}$ Le français en Afrique noire. Mythes, stratégies et pratiques. Paris, L'Harmattan. 
FCI (35) Awa se rafraîchit la mémoire / Awa rafraîchit sa mémoire

(36) Ali se ruine la santé / Ali ruine sa santé.

La phrase suivante, relevée chez J.-M. Adiaffi atteste ce type de variantes :

FCI (37a) Les gardes eux-mêmes s'étouffaient à force de pincer leur nez. (Car 71)

Nous pouvons aussi avoir, en français de Côte d'Ivoire :

p. 163

FCI (38a) Yao croise (ses / les) bras

(39a) Yao croise (ses / les) doigts.

En français de référence, les structures avec le possessif de ces paires sont plus rares :

Fréf (37b) *Guy pince son nez/ Guy se pince le nez

(38b) Guy croise (* ses / les) bras / Guy se croise les bras

(39b) Guy croise (* ses / les) doigts / * Guy se croise les doigts.

La préférence pour la structure avec le déterminant possessif son / leur sur la structure avec le déterminant défini se remarque aussi dans le cas du se réciproque :

FCI (40) Les fils présentés hier comme ennemis ont fait leur baiser de paix. (FM 31.10.97).

\section{L'apport des réponses épi et métalinguistiques aux tests psycholinguistiques}

Comme le fait remarquer Floquet (2018), les intuitions métalinguistiques des locuteurs enrichissent de façon unique la description grammaticale. L'auteur se penche sur les réponses de trois groupes de collégiens (Niamey, Toulouse et Rome) à propos d'un exemple atypique avec laver, le déterminant défini, un nom de partie du corps, mais sans le datif se : L'enfant ne lave pas le visage. Le contexte de cette question est le Test d'habileté métalinguistique "THAM-2", protocole d'enquête élaboré par Pinto \& El Euch (2015 : 65-149) pour la tranche d'âge de 9 à 14 ans. Le THAM-2 réunit plusieurs épreuves visant à mesurer la connaissance implicite de la langue et la capacité à verbaliser ses choix, en l'occurrence au niveau morphosyntaxique. Le chercheur observe non seulement le jugement épilinguistique d'un certain input mais aussi comment l'informateur justifie son choix (niveau métalinguistique) ; cela lui donne la possibilité d'analyser et de comparer simultanément les réponses épi et métalinguistiques (Boutin \& Floquet à paraitre).

La phrase L'enfant ne lave pas le visage est le dixième item sur treize de la troisième épreuve («Acceptabilité ») du THAM-2. Après s'être prononcés sur la correction de la phrase en question, les collégiens doivent expliciter leur choix, puis proposer une phrase meilleure. S'ils ont jugé l'input correct, ils n'ont pas plus de réponses à faire. Floquet (2018) constate un décalage

p. 164

entre les réponses des Niaméens d'une part (48\% jugent la phrase correcte), et des Toulousains et Romains d'autre part (respectivement $20 \%$ et $17 \%$ jugent la phrase correcte). Sachant que la distinction entre laver et se laver est bien attestée dans les français d'Afrique, l'auteur écarte d'emblée l'hypothèse de la porosité des diathèses qui ferait que se laver équivaut à laver pour les collégiens qui acceptent L'enfant ne lave pas le visage. Il recherche une explication plutôt dans une relation de commutation entre le et son (visage), ce qui déplace la question à la valeur du déterminant. Alors que les Niaméens interprètent le visage comme celui de l'enfant, aucun Toulousain n'a interprété le visage comme équivalant à son visage mais toujours le visage d'un autre. 
Nous confirmons que les constructions du type laver ne sont pas équivalentes à se laver en Côte d'Ivoire, à l'aide de trois exemples de nouchi ${ }^{6}$ avec se djèguè son équivalent :

nouchi (41) Le soir on rentre chez nous, bon on se djèguè, on daba tout tout (PhonLex-GV ${ }^{7}$ )

(42) Djekalou saint si

Type: Verbe, Synonyme(s): Se Djèguè, se Gboro

Définition: Se laver, prendre une douche

Exemple: Hier nuit j'ai tue cabris. Je m'en vais me djekalou saint si.

(nouchi.com Djekalou)

(43 Fo djêguê conhan... "Il faut la nettoyer comme ça, de cette façon."

(nouchi.com Cohan)

La construction réfléchie se djèguè est synonyme de se laver, mais djèguè peut aussi être utilisé dans une construction transitive (Faut djèguè comme ça). En outre, la question de l'acceptation en français du Niger ou d'autre pays d'Afrique de l'ouest peut être éclaircie avec des résultats au même test (THAM-2) de collégiens d'Abidjan.

p. 165

L'enquête ivoirienne du THAM-2 a été menée dans sa première phase en 2018 au collège Etimoé Makoré à Abidjan Cocody Abatta $^{8}$ auprès de 43 collégiens de 11 à 14 ans, de langue première française, répartis en plusieurs groupes. Le protocole prévoit une familiarisation avec le test, avec un entrainement oral collectif à des questions type, et comme dans les autres points d'enquête (Floquet 2018), il a été expliqué aux collégiens qu'il ne s'agissait pas d'un test scolaire et qu'ils ne subiront pas une évaluation de type scolaire. Deux épreuves du THAM-2 ont été données : «Acceptabilité » et «Ambigüité », comportant respectivement treize et sept items, parmi lesquels deux mettent en jeu des noms inaliénables en position objet : L'enfant ne lave pas le visage et Faire ses cheveux.

Le but premier du THAM-2 est d'évaluer deux niveaux d'analyse des participants : le niveau épilinguistique par des questions demandant de juger correctes ou incorrectes des phrases données, et le niveau métalinguistique par des questions demandant d'argumenter leur choix. L'un des avantages de ce protocole pour le linguiste est mettre en regard un jugement émis face un certain input (niveau épilinguistique) et une (re)catégorisation des notions linguistiques dans la justification du jugement (niveau métalinguistique). En (44) par exemple, le psycholinguiste évaluera optimale la réponse épilinguistique (a) puisqu'elle montre que le sujet a repéré une erreur dans la phrase (selon la norme de référence), et optimales aussi les réponses métalinguistiques (b, $\mathrm{c}, \mathrm{d}$,) puisqu'il a manié une recatégorisation de la notion linguistique de possession :

(44) 3.10) L'enfant ne lave pas le visage

a) C'est correct ou pas ?

$\mathrm{R}:$ ' 'est incorrect

b) Si c'est incorrect, où est l'erreur ?

$\mathrm{R}$ : l'erreur se trouve au niveau de la possession

\footnotetext{
${ }^{6}$ Le nouchi est l'argot ivoirien, multiforme, très répandu dans la société. Le site nouchi.com abrite un dictionnaire interactif d'où sont tirés les exemples (42-43). Djekalou saint si dérive de djèguèldjèkè (laver), lui-même emprunté au manding jege (poisson).

${ }^{7}$ Le projet PhonLex (« de la Phonologie aux formes Lexicales »), dirigé par Jacques Durand, Bernard Laks, JeanPierre Chevrot et Christiane Soum-Favaro a eu pour but d'approfondir plusieurs dimensions de la liaison en français. L'extrait (41) du corpus de Côte d'Ivoire a été enregistré en 2004, auprès d'un gardien de voitures à Abidjan Plateau. ${ }^{8}$ L'enquête a été dirigée par l'auteure de ce texte, assistée de Adjé Attouho Céline et Sangan Amenan Edwige. Nous remercions Edmonde Yéo Ouindé la directrice du collège et le personnel qui l'a facilitée.
} 
c) Si tu penses que c'est incorrect, comment peut-on la corriger ?

$\mathrm{R}$ : On peut la corriger en mettant « se » après ne

d) Pourquoi c'est correct comme ça ?

$\mathrm{R}$ : c'est correct comme ça car cela exprime la possession «c'est son visage qu'il ne lave p. 166 pas » (12 ans, garçon, $\left.4^{\mathrm{e}}\right)$

Pour le linguiste, ces réponses sont particulièrement intéressantes parce qu'elles donnent accès en parallèle, d'une part, à l'intégration et la restitution par le sujet de la norme de référence (l'actuation du datif $s e$ ) et, d'autre part, à la préférence du sujet pour la construction sans ce datif dans sa propre reconstitution d'une phrase correcte.

Le deuxième item retenu n'offre pas autant d'argumentation. Pinto \& El Euch (2015) prévoient une évaluation épilinguistique optimale si le sujet répond négativement à a) et une évaluation métalinguistique selon l'habileté du sujet à paraphraser les trois phrases. Ces deux habiletés sont optimales dans :

(45) 4.1.C) Faire les courses. Faire ses cheveux. Faire le clown.

a) Est-ce que le mot faire a le même sens dans les trois cas ou pas ?

$\mathrm{R}$ : Non.

b) Essaie de dire ces trois phrases différemment. Par exemple : « Faire les courses ». [...]

c) Quelle est l'autre façon de dire « Faire ses cheveux »?

$\mathrm{R}$ : Avoir une bonne coiffure en se tressant, nattant. (12 ans, garçon, $6^{\mathrm{e}}$ )

Dans le cadre de cette recherche, nous relevons uniquement la forme de la réponse donnée en c), en l'occurrence une construction avec le datif sans complément direct.

Notre question de recherche concerne les procédés linguistiques mis en jeu pour la récupération du référent comme une partie du corps du sujet de la phrase. A l'item 3.10, sur les 43 participants, 11 ont jugé la phrase correcte et 32 l'ont jugé incorrecte. Parmi ces 32 correcteurs, 14 ont proposé en correction L'enfant ne lave pas son visage ; 13 ont proposé L'enfant ne se lave pas le visage, 2 ont proposé les deux phrases, un a proposé L'enfant ne lave pas le visage de son frère, et 2 n'ont pas répondu. 29 correcteurs sur 43 ont donc interprété que 3.10 concernait le visage de l'enfant.

On peut se demander pourquoi dans l'enfant lave le visage, aucun collégien toulousain n'a pensé qu'il pouvait s'agir du visage de l'enfant, alors qu'au moins $67 \%$ des collégiens ivoiriens (au moins 29/43) ont interprété la phrase ainsi, tout comme la moitié des Niaméens. Les tests montrent que la question ne se pose pas de façon si précise chez des locuteurs non experts qui doivent répondre à une question large sur les motifs de plausibilité d'une phrase comme l'enfant lave le visage. Cependant, les commentaires de ces locuteurs non experts apportent un certain éclairage. Parmi les correcteurs qui ont privilégié le datif on a des réponses en 3.10.c) comme :

(46) se exprime la possession

le visage lui appartient

Parce qu'on sait c'est quel visage il lave

Parce que se pronom montre que c'est lui son visage qui (sic) ne lave pas.

Les correcteurs qui privilégient le déterminant possessif insistent plutôt sur l'emphase mise sur le possesseur :

(47) le visage de l'enfant et pas un autre

avec le on ne sait pas à qui appartient le visage

L'enfant lave son visage pas un visage comme ça donc on ne peut pas mettre "le visage ». Un sujet qui a donné les deux possibilités explique : 
(48) C'est correct car c'est un peut (sic) plus en rapport avec lui (13 ans, fille, $\left.5^{\mathrm{e}}\right)$.

Le protocole ne prévoit pas de demander aux sujets qui ont trouvé la phrase d'input correcte de le justifier, mais l'un d'eux a donné une explication spontanément :

(49) Parce que le visage est un (sic) partie du corps humain. Donc dans un contexte ou une personne parle de l'enfant on peut le dire (14 ans, fille, $\left.4^{\mathrm{e}}\right)$.

Nous retenons de ces diverses catégories de réponses que les collégiens sollicités ont globalement intégré les deux normes : celle du français de référence officiellement enseigné au collège Etimoé Makoré, et la norme locale pour laquelle trois constructions sont sémantiquement équivalentes et acceptables : l'enfant lave le visage (Voir aussi (29)) - l'enfant lave son visage (Voir aussi (34)) - l'enfant se lave le visage (Voir (2)) .

Uniquement 35 participants étaient présents au test «Ambigüité » du THAM-2 ; tous avaient pris part précédemment à l'épreuve «Acceptabilité ». A l'item 4.1.C, 14 collégiens ont donné comme équivalent de faire ses cheveux un complément direct introduit par un déterminant possessif :

(50 tresser ses cheveux (6)

coiffer ses cheveux (2)

parer, couper, attacher, peigner, préparer ses cheveux (1)

changer sa coupe de cheveux (1)

13 collégiens ont donné un équivalent avec le réfléchi se sans complément direct :

p. 168

(51) se coiffer, se tresser, se natter, se peigner

2 collégiens ont donné un verbe avec le datif et un complément direct :

(52) se tresser les cheveux

se faire les cheveux.

Les 6 autres réponses ne peuvent pas être validées d'un point de vue métalinguistique : 1 collégien a donné paigne les cheveux (sic), 2 n'ont changé ni le verbe ni le possessif, et 3 n'ont donné aucune réponse.

On constate que, sans qu'aucune amorce de la construction avec le datif et un complément direct ne soit faite, les collégiens ont proposé majoritairement des équivalents sans cette construction (27/29). Ces équivalents se distribuent de façon égale entre un verbe réfléchi intransitif (13) et une construction avec un verbe transitif direct et un déterminant possessif pour l'objet (14).

\section{Conclusion}

Nous avons d'abord mis en perspective plusieurs analyses du nom inaliénable (notamment une partie du corps) et du nom possédé en position objet lorsque le nom sujet correspond au possesseur, la personne humaine totale. Ces analyses éclairent le lien plus ou moins essentiel entretenu entre le nom inaliénable ou possédé et le nom possesseur. Elles éclairent aussi la prise en compte par la langue de référence de la possibilité de considérer une certaine autonomie des parties du corps ou entités inaliénables ou constitutives, pour réaliser certaines actions et non d'autres.

Le français de référence, d'une part, évite la redondance du déterminant possessif pour des noms possédés lorsque le datif est présent, mais aussi, sans ce datif, lorsque la partie du corps est considérée comme ayant une certaine autonomie pour réaliser l'action, ou pouvant décentraliser en partie le rôle de l'agent. Dans d'autres langues au contraire, le lien essentiel ou inséparable entre la partie du corps et la personne totale est obligatoirement explicité. 
En français de Côte d'Ivoire, plusieurs processus cognitifs se mêlent du fait de la concurrence des normes et des systèmes linguistiques. Nous avons fait deux observations principales concernant les constructions avec un nom inaliénable en position de complément verbal direct. D'une part, l'extension générale du déterminant défini pour la valeur spécifique, ou non générique, couvre en partie ces cas des noms inaliénables. D'autre part, l'extension parallèle du déterminant possessif recouvre à la fois les noms possédés et inaliénables, les constructions avec ou sans datif, et les divers types d'action réalisée.

p. 169

Dans les cas de présence du déterminant défini, il ne nous semble pas opportun de faire porter l'interprétation possessive sur celui-ci ni sur un processus systématisé à partir des propriétés syntaxiques de l'objet ou des propriétés sémantiques du verbe. L'interprétation de la relation entre le sujet possesseur et l'objet possédé est quasi unanime et elle repose plus sur une inférence : l'évidence du lien essentiel ou inséparable entre la partie du corps et la personne totale. Dans ces cas, la possession n'est, tout simplement, pas explicitée par le discours.

Lorsque les locuteurs ne suivent pas la norme du français de référence mais celle du français de Côte d'Ivoire, le datif disparait et le déterminant défini comme le déterminant possessif sont tous deux susceptibles d'apparaitre auprès du nom possédé ou inaliénable, sans que l'interprétation soit gênée. Le déterminant possessif explicite la relation, d'appartenance ou d'inclusion, comme dans les langues ivoiriennes, alors que le déterminant défini la laisse implicite.

Le français ivoirien a les propriétés d'une langue hybride dans la mesure où on y observe des traits régis par des normes diverses : celle du français de référence et d'autres du français de Côte d'Ivoire et des langues ivoiriennes. En outre, il n'est pas toujours possible d'attribuer à une construction une origine à l'une de ces langues étant donné leur variabilité interne et leurs points communs. Les propriétés des langues d'une même aire plurilingue sont très mêlées dans l'innovation d'une construction nouvelle qui, tout en ressemblant à plusieurs de ces langues, peut n'être totalement identique à aucune. L'hybridation aboutit à un ensemble entièrement nouveau et autonome vis-à-vis de ses sources. Enfin, cet ensemble nouveau est lui-même porteur aussi d'une grande variabilité interne.

\section{Bibliographie}

Bally, Charles, 1926. L'expression des idées de sphère personnelle et de solidarité dans les langues indo-européennes : Festschrift Gauchat, Aarau (Sauerländer), p. 68-78.

Boutin, Béatrice Akissi, 2002. Description de la variation: Etudes transformationnelles des phrases du français de Côte d'Ivoire. Thèse de doctorat. Université de Grenoble 3. Villeneuve d'Ascq : Presses Universitaires du Septentrion.

Creissels, Denis, 2006. Syntaxe générale, une introduction typologique, tommes 1 et 2. Paris : Hermès/Lavoisier.

Creissels, Denis, 2004. Cours de Syntaxe de Sciences Du Langage, Université Lyon 2. Document non publié constitué de 32 chapitres.

Floquet, Oreste, 2018. Remarques épilinguistiques et métalinguistiques sur l'expression de la possession inaliénable à partir de réponses à un test d'acceptabilité (Niamey, Toulouse, Rome). In Floquet, O. (éd) Aspects linguistiques et sociolinguistiques des français africains, p. 35-52, Roma : Sapienza Università Editrice.

Guéron, Jacqueline, 1983. Emploi 'possessif' de l'article 'défini' en français, Langue française $\mathrm{n}^{\circ}$ 58 : 23-35, Paris : Larousse. 
Jabet, Marita, 2003. Caractéristiques de référenciation dans le français abidjanais. La référence aux entités : les cas de déterminants et de pronoms sujet (licentiatavhandling). PERLES (Petites Études Romanes de Lund Extra Seriem) n ${ }^{\circ}$ 17. Lund : Institut d'Études Romanes.

Junker, Marie-Odile \& Martineau, France, 1987. Les possessions inaliénables dans les constructions objet. Revue Romane, ${ }^{\circ} 22,2$, p. 194-209.

Kouadio N'Guessan Jérémie, 1977. L'enseignement du français en milieu baoulé, problèmes des interférences linguistiques et socioculturelles, thèse de 3e cycle, Université de Grenoble 3.

Kouadio N'Guessan Jérémie, 1999. Quelques traits morphosyntaxiques du français écrit en Côte d'Ivoire, Cahiers d'études et de recherches francophones, Langues, Vol. II, nº 4 : 301-314, Paris : AUPELF - UREF

Kouamé Koia Jean-Martial, 2013. Les classes ivoiriennes entre monolinguisme de principe et plurilinguisme de fait. In Danielle Omer et Frédéric Tupin, Éducations plurilingues. L'aire francophone entre héritages et innovations, Rennes : Presses Universitaires de Rennes, 167179.

Knutsen, Anne Moseng, 2007. Le français à Abidjan (Côte d'Ivoire). Vers une analyse multidimensionnelle de la variation, Le français en Afrique, $\mathrm{n}^{\circ} 22$, p. 127-159.

Kpli, Jean-François, 2014. Syntaxe des possessions inaliénable et aliénable : approche métaopérationnelle, Multilinguales, 3 [En ligne]

Lamiroy, Béatrice \& Delbecque, Nathalie, 1997 : «The possessive dative in Romance and Germanic languages », in W. van Belle et W. van Langendonck (éds.) The Dative, Theoritical and Constrastive Studies, Vol. 2 : Case and Grammatical Relations across Languages, p. 29-74. Amsterdam / Philadelphia : John Benjamins.

Pinto, Maria Antonietta \& El Euch, Sonia, 2015. La conscience métalinguistique - théorie, développement et instruments de mesure. Laval, PUL.

Prignitz, Gisèle, 2005. Observations sur un corpus récent recueilli à Ouagadougou, Le français en Afrique, $\mathrm{n}^{\circ} 21,193-210$.

Riegel, Martin, 1991. Transitivité et conditionnements cognitifs : la relation partie-tout et complémentaire verbale. Linx, $\mathrm{n}^{\circ}$ 24, Sur la transitivité dans les langues, p. 133-146.

Tymian, Judith, Kouadio N'Guessan Jérémie, \& Loucou, Jean-Noël. 2003. Dictionnaire baoulé français, Abidjan : NEI. 